Technical Note

\title{
Automated MTF measurement in CT images with a simple wire phantom
}

\author{
Choirul ANAM ${ }^{1, a}$, Toshioh FUJIBUCHI ${ }^{2}$, Freddy HARYANTO ${ }^{3}$, Wahyu Setia BUDI ${ }^{1}$, Heri SUTANTO ${ }^{1}$, Kusworo AdI ${ }^{1}$, \\ Zaenul MUHLISIN ${ }^{1}$, Geoff DOUGHERTY ${ }^{4}$ \\ ${ }^{I}$ Department of Physics, Faculty of Mathematics and Natural Sciences, Diponegoro University, Jl. Prof. Soedarto SH, \\ Tembalang, Semarang 50275, Central Java, Indonesia \\ ${ }^{2}$ Department of Health Sciences, Faculty of Medical Sciences, Kyushu University, 3-1-1 Maidashi, Higashi-ku, Fukuoka $812-$ \\ 8582, Japan \\ ${ }^{3}$ Department of Physics, Faculty of Mathematics and Natural Sciences, Bandung Institute of Technology, Ganesha 10, \\ Bandung 40132, West Java, Indonesia \\ ${ }^{4}$ Department of Applied Physics and Medical Imaging, California State University Channel Islands, Camarillo, CA 93012, \\ USA \\ ${ }^{a}$ E-mail address: anam@fisika.undip.ac.id
} (received 7 March 2019; revised 28 July 2019; accepted 30 July 2019)

\begin{abstract}
This study developed a simple wire phantom and an algorithm to automatically measure the modulation transfer function (MTF) in computed tomography (CT) and implemented it to evaluate the effect of focal spot size and reconstruction filter type. The phantom consisted of a resin cylinder filled with water, with a tin wire of diameter 0.1 $\mathrm{mm}$ positioned along the center of the cylinder. The automated MTF algorithm used an axial image of the phantom and comprised several steps. The center position of a region of interest (ROI) was automatically determined at the center of the wire image. The pixels were then summed along the y-direction to obtain the profile of the pixel values at a point along the $\mathrm{x}$-direction. Following this, both edges of the profile were made equal to zero. The profile curve was then normalized so that the total of all the data was equal to unity. The normalized profile curve is the line spread function (LSF), and the MTF curve was obtained by taking its Fourier transform. Our system (phantom and algorithm) is able to differentiate the MTFs of CT images from different focal sizes and reconstruction filter types.
\end{abstract}

Key words: modulation transfer function (MTF); spatial resolution; simple phantom; image quality; CT scan.

\section{Introduction}

Computed tomography (CT) has become an important tool for medical imaging [1]. Its use for medical purposes has increased rapidly worldwide [2], especially after the advent of helical CT scanning [3] and multi-detector row CT [4]. With the increasing effectiveness and efficiency of CT scanners, their complexity has also increased. Many input parameters, directly and indirectly, affect image quality [5,6]. CT performance should be evaluated holistically and quality control should be performed on a regular basis. Among the image quality parameters to be monitored are (high contrast) spatial resolution $[7,8]$. The simplest way to characterize the spatial resolution is to use a bar pattern [9], although this approach is biased by observer subjectivity. A more objective description is obtained by using the modulation transfer function (MTF) curve [10]. The MTF curve of a CT image provides information across all spatial frequencies of the image [11-14].
It is calculated using the Fourier transform of the line spread function (LSF), point spread function (PSF) or edge spread function (ESF), and it can also be estimated directly using a phantom with a series of bar patterns $[15,16]$.

Obtaining MTF from CT images is complicated and is influenced by many parameters, such as the type of phantom (e.g. wire, bead, edge) $[10,11,17,18]$ and material of the object (e.g. bone, acrylic, polyethylene, stainless steel, etc) [17], and by CT scanner parameters, such as field of view (FOV) [19], image reconstruction kernels [20], slice thickness [17], focal spot size [21], single or dual slice CT [22], and different image reconstruction algorithms [23-25]. The details of measurement, e.g. distance to iso-center [17,26], xy and radial direction [11], noise magnitude desired [27], ROI size [18,28], and whether curve fitting is used [17,29] are also important. During measurement should be kept constant except the parameter of interest. 
Measurement of spatial resolution using MTF generally uses a specific standardized phantom, such as the ACR Accreditation Phantom (Gammex, Middleton, WI, USA) [30], AAPM CT Performance Phantom (CIRS, Norfolk, VA, USA) [31], or Catphan Phantom (Phantom Laboratory, Salem, NY, USA) [32]. Recently, one study reported that the MTF curve could be measured using the edge of a head PMMA phantom [19]. However, these phantoms may not be available in every CT center, especially in developing countries. For this reason, we have developed a simple phantom using readily available materials, and simple software to automatically calculate MTF, for use as part of quality assurance programs for CT centers that do not have standard equipment. We used our system to investigate the MTFs from different focal spot sizes and various reconstruction filter types.

\section{Methods}

\section{Simple wire phantom}

We constructed a simple self-built wire phantom from readily available material to measure MTF, as shown in Figure 1. The phantom was made from a $200 \mathrm{ml}$ CT injector syringe (Kyorindo Nemoto Ltd., Japan) with a diameter (D) of $4.8 \mathrm{~cm}$. A thin wire with a diameter (d) of $0.1 \mathrm{~mm}$ and a length (L) of about $5 \mathrm{~cm}$ was positioned at the center of the resin cylinder along an axis perpendicular to the axial plane. If a diameter of $0.1 \mathrm{~mm}$ is not available, a diameter between $0.1-0.2 \mathrm{~mm}$ could be used [18]. Finally, the phantom was filled with tap water to a volume of about $150 \mathrm{ml}$, although this is optional. It can also be left filled with air [18]. This phantom is very easy to be made and very cheap.

\section{Software for MTF measurement}

Figure 2 shows the flow chart to automatically calculate the MTF using MatLab (Mathworks). The center position of the region of interest (ROI) was at the center of the wire image and it was automatically determined. It was carried out by thresholding using the threshold value of $+100 \mathrm{HU}$, chosen based on the fact that the value of HU for a tin wire is around $+400 \mathrm{HU}$ and the water around a tin wire is around $0 \mathrm{HU}$. After thresholding, a center point was determined using the centroid equation.

$\left(x_{c}, y_{c}\right)=\frac{1}{N} \sum_{i=1}^{n} \sum_{j=1}^{n}\left(x_{i}, y_{j}\right)$

Eq. 1

The ROI was $32 \times 32$ pixels, and the image was cropped to that size. Then, the pixels were summed along the $y$-direction to obtain the profile of the pixel values at a point along the $\mathrm{x}$ direction $(S(x))$.

$S(x)=\sum_{y} R O I(x, y)$

Eq. 2

$\operatorname{ROI}(x, y)$ is the pixel value at a location $(\mathrm{x}, \mathrm{y})$ in the region of interest. Following this, both edges of the profile were made equal to zero $\left(S^{\prime}(x)\right)$. The zeroing process was performed automatically by calculating the mean value of 5 pixels on the left and 5 pixels on the right end of $S(x)$, using Equation 3.

$\overline{S\left(x_{5}\right)}=\frac{\sum_{i=1}^{5} S\left(x_{i}\right)+\sum_{i=n-5}^{n} S\left(x_{i}\right)}{10}$ Eq. 3

All points on $S(x)$ were then reduced by this mean value.

$S^{\prime}(x)=S(x)-\overline{S\left(x_{5}\right)}$

Eq. 4

Next, the curve was normalized so that the total of all data was equal to unity and the MTF had a value of 1.0 at zero spatial frequency [9]. The normalized $S^{\prime}(x)$ curve is the LSF. The process was implemented by dividing all points on the curve $S^{\prime}(x)$ by the total value of $S^{\prime}(x)$

$\operatorname{LSF}(x)=\frac{S^{\prime}(x)}{\sum_{i=1}^{n} S^{\prime}\left(x_{i}\right)}$

The $\mathrm{x}$-axis in the LSF curve was then converted from pixel number to distance in $\mathrm{mm}$ (or $\mathrm{cm}$ ), using the distance between pixels extracted from the DICOM header and setting the central position of the LSF to zero. Finally, the MTF curve that describes the spatial resolution of the image was obtained by the Fourier transform of the LSF curve [33].

$\operatorname{MTF}(f)=|F(\operatorname{LSF}(x))|=\left|\int_{-\infty}^{+\infty}\left[\operatorname{LSF}(x) e^{-2 \pi j x f}\right] d x\right| \quad$ Eq. 6

where $f$ denotes the spatial frequency and $\mathbf{F}$ indicates the Fourier transform.

(a)

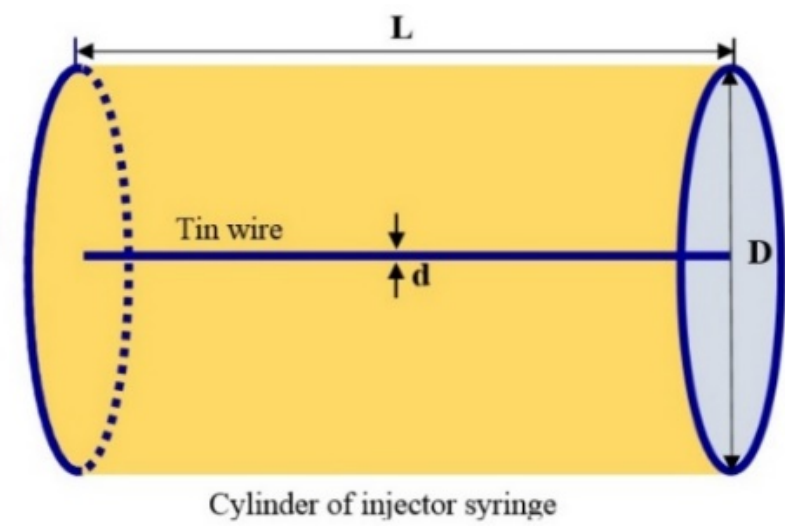

(b)

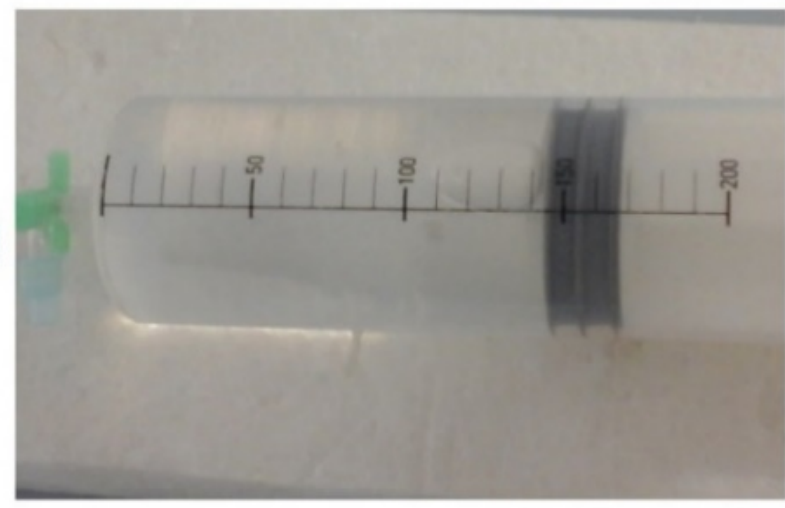

Figure 1. (a) A schematic diagram of the wire phantom for MTF measurement, and (b) Photograph of the developed phantom. 


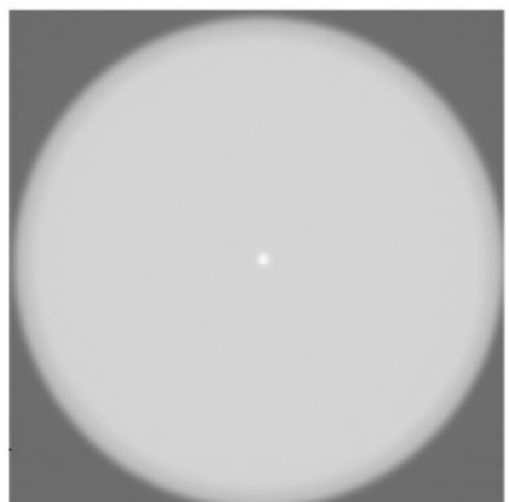

Image of phantom
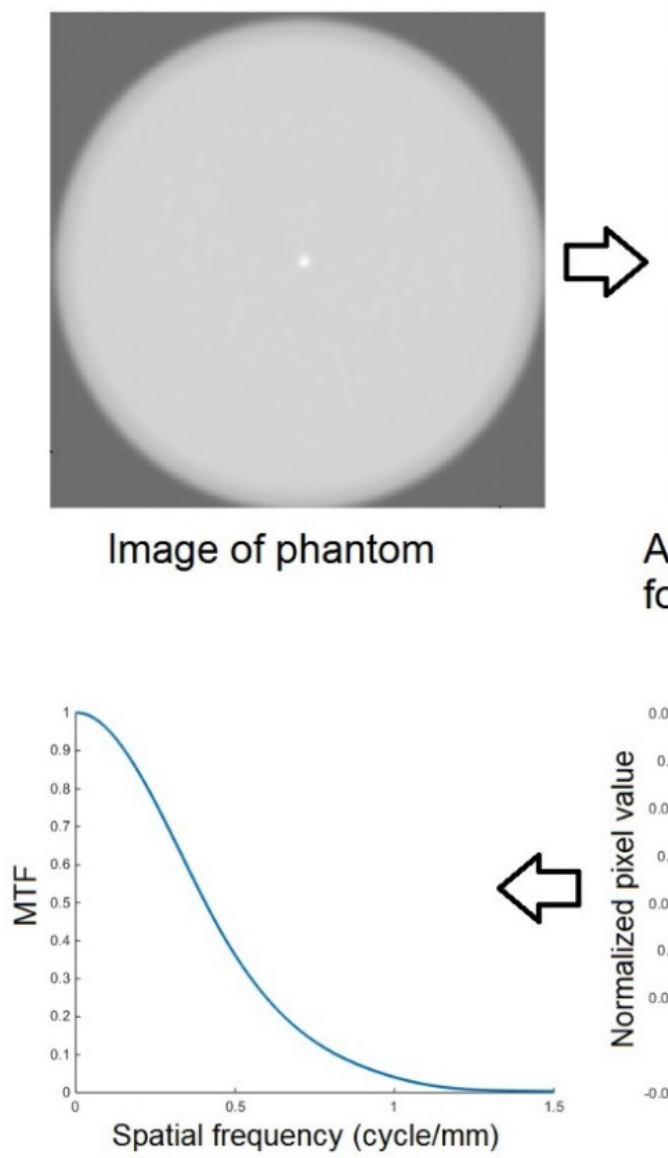

Automated ROI determination for MTF calculation
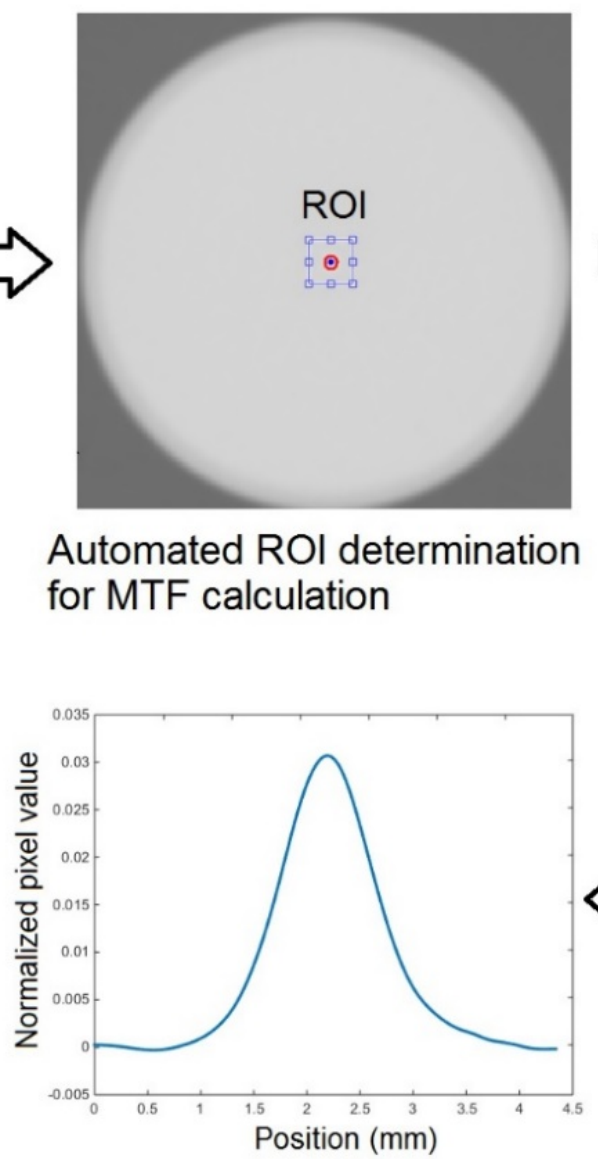

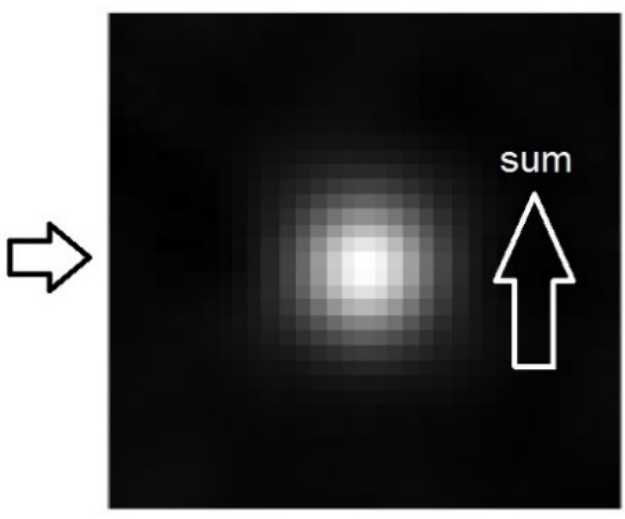

Cropped image

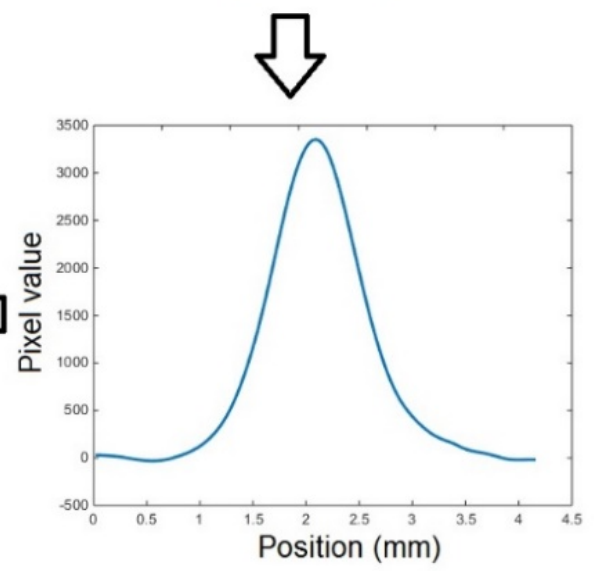

Figure 2. Processes for calculating the MTF from the phantom.

\section{Validation and implementation of MTF measurement}

The MTF phantom was used to evaluate the spatial resolution of a new 4-row multi-slice CT scanner Alexion ${ }^{\mathrm{TM}}$ installed at the Department of Health Sciences, Faculty of Medical Sciences, Kyushu University, Japan.

The automated MTF method was validated using the standard fitting method proposed by Yin et al. [34]. The equation for the fitted LSF was a combination of analytical functions:

$L S F(x)=a_{2} e^{\left[-0.5\left(\frac{x-a_{1}}{a_{3}}\right)^{2}\right]}+a_{4} e^{\left[-\frac{\left|x-a_{1}\right|}{a_{5}}\right]}$

Eq. 7

where $a_{1}$ denotes the center of fitted LSF, $a_{2}$ represents the weighting factor of the Gaussian function, $a_{3}$ denotes the standard deviation of the Gaussian function, $a_{4}$ represents the weighting factor of the exponential function, and $a_{5}$ denotes the slope of the exponential function. This fitted function is applicable to a non-normalized LSF. The parameters of $a_{1}, a_{2}$, $a_{3}, a_{4}$ and $a_{5}$ were chosen by trial and error so that the curve fits to a measured LSF. After the parameters were already chosen then the MTF is calculated using the equation:

$\operatorname{MTF}(f)=\frac{\sqrt{2 \pi} a_{2} a_{3} e^{\left[-2 \pi^{2} a_{3}^{2} f^{2}\right]}+2 a_{4} a_{5} /\left(1+4 \pi^{2} a_{5}^{2} f^{2}\right)}{\sqrt{2 \pi} a_{2} a_{3}+2 a_{4} a_{5}}$

Eq. 8 where $f$ is spatial frequency. This MTF equation has been normalized to provide unity at zero frequency. The percentage difference between MTF $50 \%$ of the automated method and the fitting method will be compared.

In this study, our system (phantom and software) was implemented to evaluate the effect of focal spot sizes of 1.1 $\mathrm{mm}$ and $1.7 \mathrm{~mm}$ on the MTF. Three types of reconstruction filter (FC13, FC52, and FC30) were evaluated. FC13 is a filter used for soft tissue, F52 for lung, and F30 for bone. The other input parameters were kept constant (tube voltage $120 \mathrm{kVp}$, rotation time $1 \mathrm{~s}$, a field of view $7 \mathrm{~cm}$, slice thickness $4 \mathrm{~mm}$ ).

\section{Results}

\section{MTF curve validation}

Our algorithm using a threshold value of $+100 \mathrm{HU}$ successfully determined the center of ROI in the center of the point image for all images used and successfully calculated MTF curves. To validate the resulting MTFs, it was compared with the standard calculation using the fitting method developed by Yin et al. [34], as shown in Figure 3. The figure shows that both MTF curves are very similar. The difference in MTF $50 \%$ for both methods is $-2.8 \%$ and $2.4 \%$ for $1.1 \mathrm{~mm}$ and $1.7 \mathrm{~mm}$ focal spots respectively. 
(a)

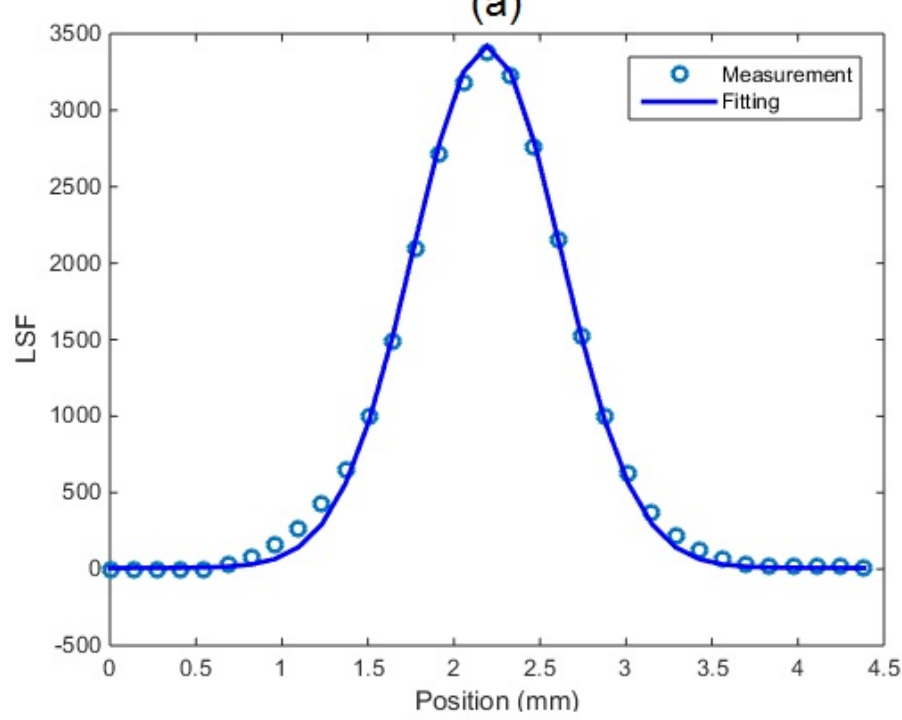

(c)

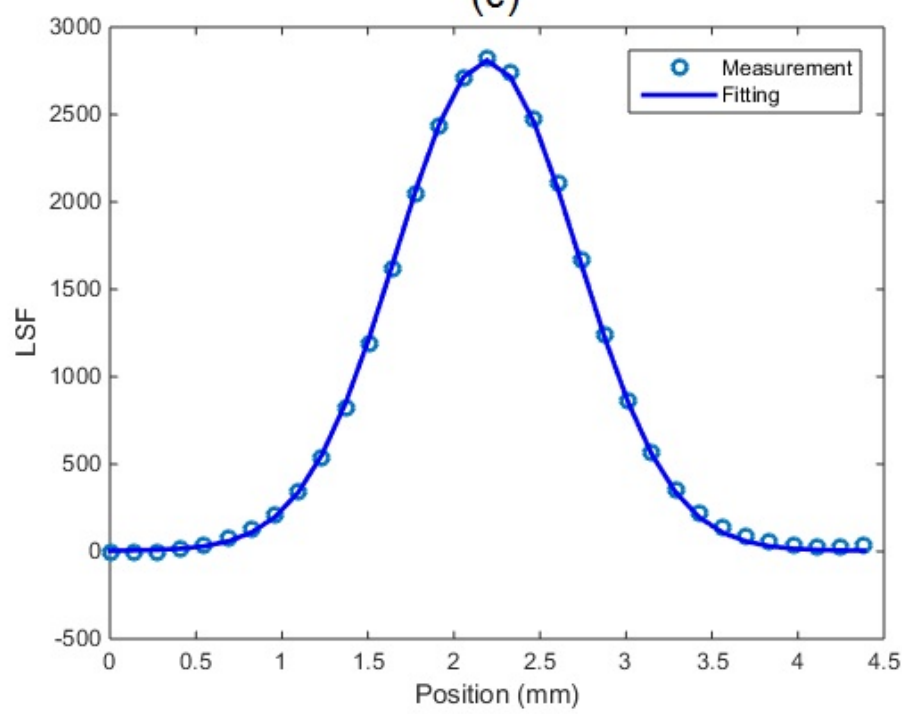

(b)

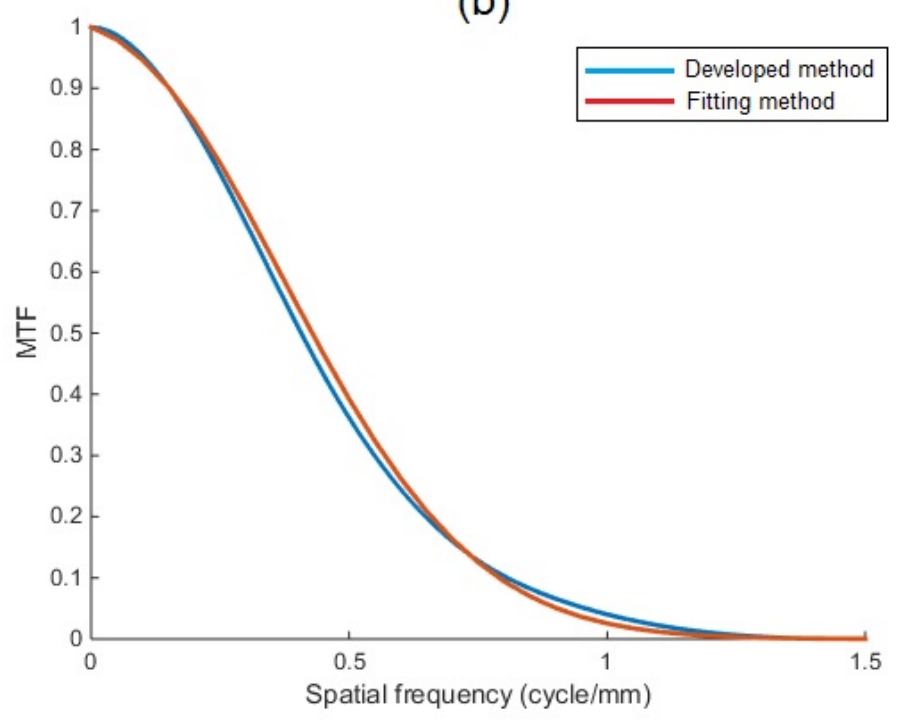

(d)

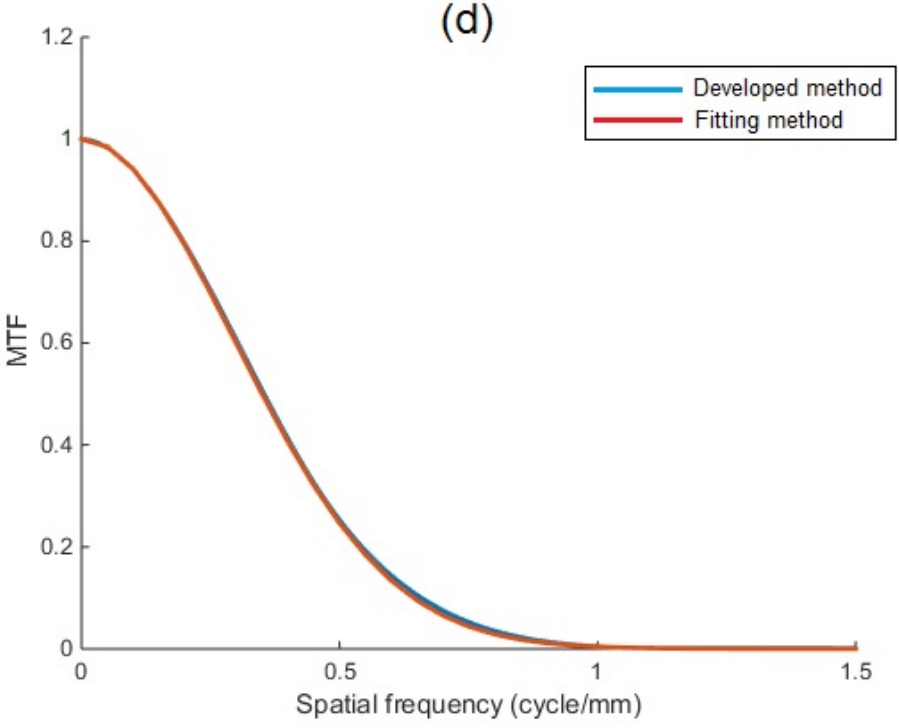

Figure 3. LSF and MTF curves calculated by automated method vs fitting method. The first column is LSF curves from measurement and fitting method, and the second column is MTF curves from automated method and fitting method. The first row is for a focal spot of $1.1 \mathrm{~mm}$ and the second row is for a focal spot of $1.7 \mathrm{~mm}$.

\section{LSF and MTF curves for different focal spot size}

The LSF and MTF curves for the focal spot size of 1.1 and 1.7 $\mathrm{mm}$ are shown in Figure 4. The first row is for FC13 filter, the second row is for FC30 filter, and the third row is for FC52 filter. The MTF 50\% values for the focal spot size of 1.1 and $1.7 \mathrm{~mm}$ are shown in Table 1. There is a clear difference in the LSF and MTF curves between spot sizes of 1.1 and $1.7 \mathrm{~mm}$. A focal spot of $1.1 \mathrm{~mm}$ produced a higher spatial resolution compared with a focal spot of $1.7 \mathrm{~mm}$, as expected.
Table 1. The MTF $50 \%$ values for various focal spot sizes.

\begin{tabular}{lcc}
\hline \hline & \multicolumn{2}{c}{ MTF 50\% (cycle/mm) } \\
\cline { 2 - 3 } & Focal spot of $\mathbf{1 . 1} \mathbf{~ m m}$ & Focal spot of $\mathbf{1 . 7} \mathbf{~ m m}$ \\
\hline FC13 filter & 0.41 & 0.36 \\
FC30 filter & 0.83 & 0.65 \\
FC52 filter & 0.93 & 0.70 \\
\hline \hline
\end{tabular}



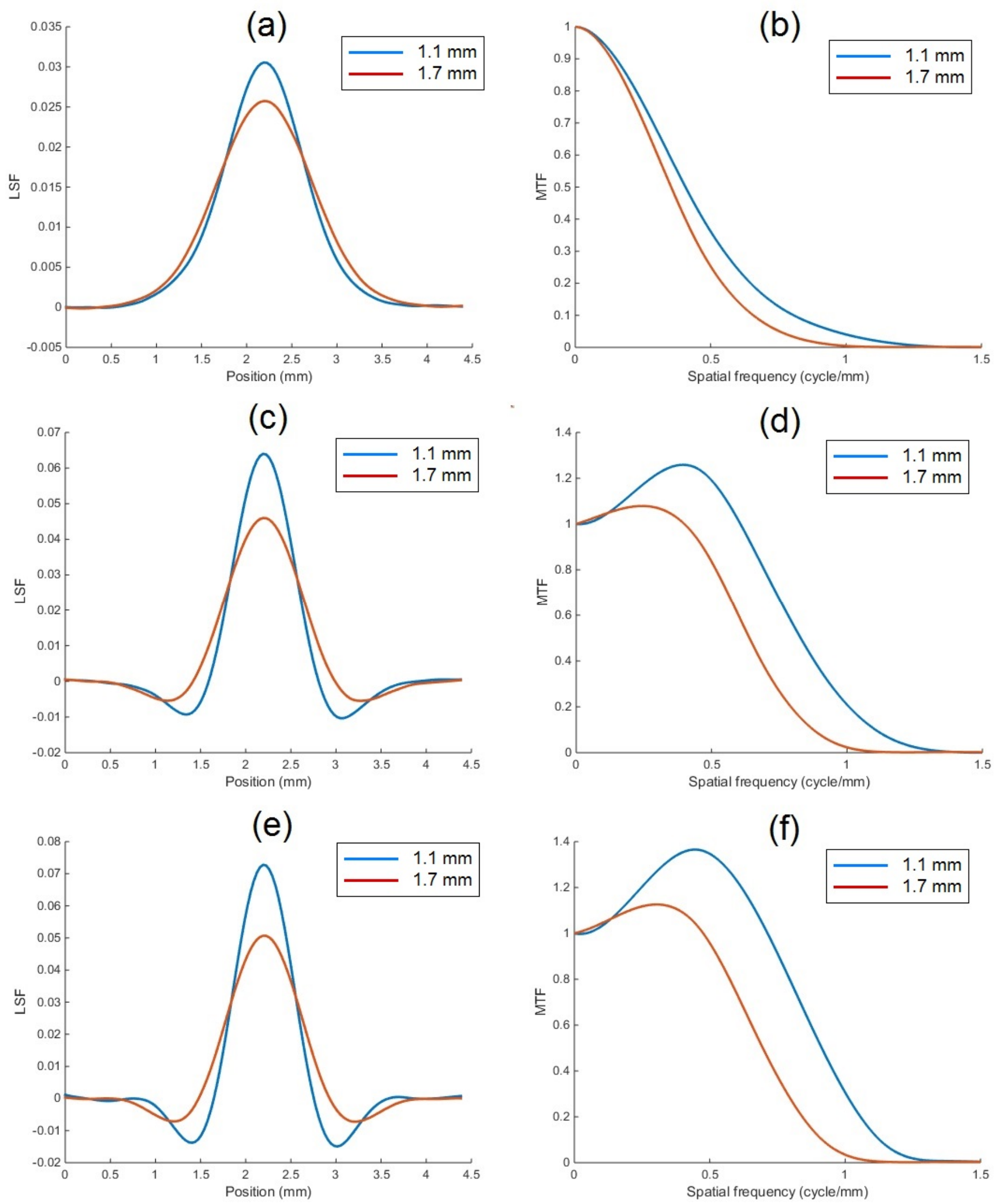

Figure 4. LSF and MTF curves for different values of the focal spot. The LSF curves are on the left, and the MTF curves are on the right. The first row is for FC13 filter, the second row is for FC30 filter, and the third row is for FC52 filter. 

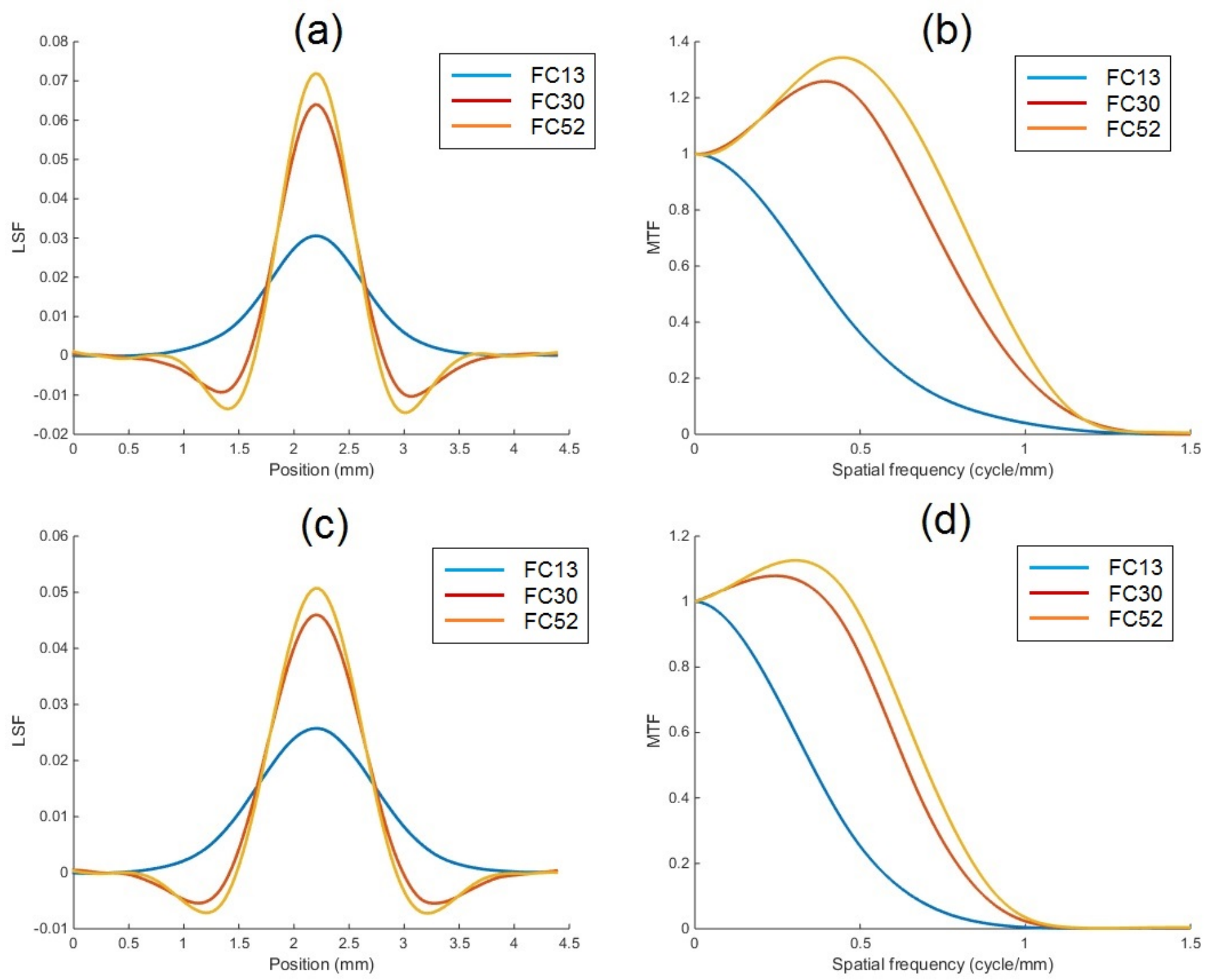

Figure 5. LSF and MTF curves for different filter: FC13, FC52, and FC30. The left is LSF curve, and the right is the MTF curve. The first row is for focal spot size of $1.1 \mathrm{~mm}$, and the second row is for focal spot size of $1.7 \mathrm{~mm}$.

\section{LSF and MTF for different reconstruction filters}

The LSF and MTF curves for three filters: FC13, FC30, and FC52 are shown in Figure 5. The first row is for focal spot size of $1.1 \mathrm{~mm}$, and the second row is for focal spot size of $1.7 \mathrm{~mm}$. The FC13 filter produced the widest LSF curves, while the other two filters (FC30 and FC52) produced narrower but similar curves, with the FC50 curves a little sharper than those from the FC30 filter. Figure 5 also shows there were no negative values in the LSF curves around the object (wire) for the FC13 filter, while the FC30 and FC52 filters produced significant negative values. The MTF curves generated by the FC13 filter had the lowest spatial resolution, followed by the FC30 filter, and the highest spatial resolution was from the FC52 filter. The MTF 50\% values for various reconstruction filters are listed in Table 2.
Table 2. The MTF 50\% values (in the unit of cycle/mm) for various reconstruction filters.

\begin{tabular}{lccc}
\hline \hline & \multicolumn{3}{c}{ MTF 50\% (cycle/mm) } \\
\cline { 2 - 4 } & FC13 & FC30 & FC52 \\
\hline Focal spot size of $1.1 \mathrm{~mm}$ & 0.41 & 0.83 & 0.93 \\
Focal spot size of $1.7 \mathrm{~mm}$ & 0.36 & 0.65 & 0.70 \\
\hline \hline
\end{tabular}

\section{Discussion}

Spatial resolution is one of the principal parameters that determine the image quality of CT images [7,35]. An objective description of spatial resolution is obtained using the MTF curve [10]. The spatial resolution should be evaluated regularly as part of a quality control program. The most common technique to determine the MTF of CT images is to use a wire phantom [7], although other types of the phantom can also be used including micro-bead with a very small diameter [7], the 
edge [16], or line pair [11]. The types of objects to determine the MTF are usually available in a special module on the standard phantom, e.g. example ACR Accreditation Phantom, AAPM CT Performance Phantom, or Catphan Phantom. The AAPM CT Performance Phantom contains a stainless steel wire with a diameter of $0.23 \mathrm{~mm}$ positioned longitudinally for MTF calculation [31]. The ACR CT Accreditation Phantom contains two small tungsten carbide beads with a diameter of $0.3 \mathrm{~mm}$ [30]. The Catphan Phantom contains two individual beads with a diameter of $0.28 \mathrm{~mm}$ and $0.18 \mathrm{~mm}$ in diameter, and a tungsten wire with a diameter of $50 \mu \mathrm{m}$ is located $6 \mathrm{~cm}$ from the center of the module. The beads and wire are used to measure LSF that can be used to calculate the MTF [10].

These phantom types are standard phantoms used for MTF measurements. However, some CT scan centers may not have one of these standard phantoms. This study has shown a simple method for developing an in-house phantom and software that is automatically used to measure MTF. The results of the automatic MTF calculation are similar to those obtained using a fitting method [34]. The difference of MTF 50\% from both approaches is less than 3\%. The automatic calculation runs in about 1 second, although the fitting method calculation is timeconsuming because changes in parameters $\mathrm{a}_{1}, \mathrm{a}_{2}, \mathrm{a}_{3}, \mathrm{a}_{4}$, and $\mathrm{a}_{5}$ are fitted by trial and error.

It should be noted that the method used in this study was to sum several pixels along a y-direction to get the LSF. It is known, that the MTF calculated with such an approach is dependent on ROI size [10]. Both our method and a standard fitting method [34] were applied to the same data (LSF from a sum of profiles) and produced similar results. This comparison shows that both algorithms calculate the same MTF from the same LSF. However, it does not prove that either MTF is correct. One way to prove that the MTF is correct has been proposed by Ohkubo et al. [36] and refined by Kayugawa et al. [10].

The spatial resolution is strongly influenced by the type of reconstruction filter as reported previously [19], and our system confirmed this phenomenon. The user should select the type of filter appropriately based on clinical purposes. The FC13 filter produces low spatial resolution and low noise, so it is suitable for soft tissue applications, which require low noise images and only moderate spatial resolution. The FC52 filter produces images with not only very high spatial resolution but also high noise, so it is suitable for the inspection of lungs, which require a very high spatial resolution but where noise is not a major consideration. The FC30 filter produces a moderate-to-high spatial resolution and noise and is suitable for bone examination.
The spatial resolution is also strongly influenced by the size of the focal spot as reported previously [21]. Again, our system was able to confirm this. The CT scanner used in this study has two focal spot sizes, $1.1 \times 1.1 \mathrm{~mm}^{2}$ and $1.7 \times 1.7 \mathrm{~mm}^{2}$. The focal spot for a tube current of 50-150 mA was $1.1 \times 1.1 \mathrm{~mm}^{2}$ and it was automatically adjusted to the larger focal spot $(1.7 \times$ $1.7 \mathrm{~mm}^{2}$ ) for a tube current of 200-300 mA. We set tube currents of $150 \mathrm{~mA}$ and $200 \mathrm{~mA}$ to get the two different tube currents. The automatic focal spot adjustment based on the tube current due to the high tube current produces a high thermal load on the X-ray target, and the system automatically re-sets to a larger focal spot size to dissipate the additional heat.

Our system is able to differentiate MTF curves from different focal spot sizes and reconstruction filter types as achieved using standard phantoms. The main limitation of this current study was that our system was performed on only one type of scanner, the MDCT scanner 4 Alexion $^{\mathrm{TM}}$. It should be noted that this phantom is an in-house phantom, so its repeatability is not guaranteed. Such in-house phantom must be compared to a standard phantom before it can be applied in a clinical setting. Our phantom is an alternative if the standard phantom is not available at a CT center. For acceptance tests, CT centers should try to employ standard phantoms. However, for constancy tests, an in-house phantom is acceptable so long as it has been compared to the standard phantom before use.

In this study, the MTF is calculated using a 1D FFT, after 2D wire images are averaged in the y-axis. The use of a 2D FFT on wire image is a more sophisticated approach for MTF measurement [37]. Although the 1D FFT is simpler than using 2D FFT for measuring MTF, its accuracy has been confirmed [10].

\section{Conclusions}

We have successfully developed a simple in-house wire phantom and software to automatically measure MTF. This system was able to differentiate MTFs from different focal spot sizes and reconstruction filter types. The developed in-house phantom may be used in the regular quality control (QC) program if the standard phantom is not available.

\section{Acknowledgments}

This work was funded by the Penelitian Sumber Dana Selain APBN Fakultas Sains \& Matematika, Universitas Diponegoro, Tahun Anggaran 2019, contract number. 4936/UN7.5.8/PP/2019. 


\section{References}

[1] Verdun FR, Racine D, Ott JG, et al. Image quality in CT: From physical measurements to model observers. Phys Med. 2015;31(8):823-843.

[2] International Atomic Energy Agency. Status of computed tomography: Dosimetry for wide cone beam scanners. IAEA-Human Health Reports No 5. 2011.

[3] Kalender WA, Seissler W, Klotz E, Vock P. Spiral volumetric CT with single-breath-hold technique, continuous transport, and continuous scanner rotation. Radiology. 1990;176(1):181-183.

[4] McCollough CH, Zink FE. Performance evaluation of a multi-slice CT system. Med Phys. 1999;26(11):2223-2230.

[5] McNitt-Gray MF. AAPM/RSNA physics tutorial for residents: topics in CT-radiation dose in CT. RadioGraphics. 2002;22(6):1541-1553.

[6] Primak AN, McCollough CH, Bruesewitz MR, et al. Relationship between noise, dose, and pitch in cardiac multi-detector row CT. RadioGraphics. 2006;26(6):1785-1794.

[7] Wilson JM, Christianson OI, Richard S, Samei E. A methodology for image quality evaluation of advanced CT systems. Med Phys. 2013;40(3):031908.

[8] Anam C, Haryanto F, Widita R, Arif I. New noise reduction method for reducing CT scan dose: Combining Wiener filtering and edge detection algorithm. AIP Conference Proceedings. 2015;1677:040004.

[9] McCollough CH, Bruesewitz MR, McNitt-Gray MF, et al. The phantom portion of the American College of Radiology (ACR) Computed Tomography (CT) accreditation program: Practical tips, artifact examples, and pitfalls to avoid. Med Phys. 2004;31(9):2423-2442.

[10] Kayugawa A, Ohkubo M, Wada S. Accurate determination of CT point-spread-function with high precision. J Appl Clin Med Phys. 2013;14(4):216-226.

[11] Friedman SN, Fung GSK, Siewerdsen JH, Tsui BMW. A simple approach to measure computed tomography (CT) modulation transfer function (MTF) and noise-power spectrum (NPS) using the American College of Radiology (ACR) accreditation phantom. Med Phys. 2013;40(5):051907.

[12] Judy PF. The line spread function and modulation transfer function of a computed tomographic scanner. Med Phys. 1976;3(4):233236.

[13] Droege RT, Morin RL. A practical method to measure the MTF of CT scanners. Med Phys. 1982;9(5):758-760.

[14] Nickoloff EL Riley R. A simplified approach for modulation transfer function determinations in computed tomography. Med Phys. 1985;12(4):437-442.

[15] Samei E, Ranger NT, Dobbins JT, Chen Y. Intercomparison of methods for image quality characterization. I. Modulation transfer function. Med Phys. 2006;33(5):1454-1465.

[16] Narváez M, Graffigna JP, Gómez ME, Romo R. Application of oversampling to obtain the MTF of digital radiology equipment. J Phys Conf Ser. 2016;705(1):012057.

[17] Takenaga T, Katsuragawa S, Goto M, et al. Modulation transfer function measurement of CT images by use of a circular edge method with a logistic curve-fitting technique. Radiol Phys Technol. 2015;8(1):53-59.

[18] Ichikawa K, Hara T, Niwa S, Ohashi K. Method of measuring modulation transfer function using metal wire in computed tomography. Nihon Hoshasen Gijutsu Gakkai Zasshi. 2008;64(6):672-680.

[19] Anam C, Fujibuchi T, Budi WS, et al. An algorithm for automated modulation transfer function measurement using an edge of a PMMA phantom: Impact of field of view on spatial resolution of CT images. J Appl Clin Med Phys. 2018;19(6):244-252.

[20] Jun-Yan R, Guo-Tao F, Cun-Feng W, et al. Measurement of spatial resolution of the micro-CT system. Chinese Physics C. 2010;34(3):412-416.

[21] Arabi H, Asl ARK, Aghamiri SM. The effect of focal spot size on the spatial resolution of variable resolution X-ray CT Scanner. Iran J Radiat Res. 2010;8(1):37-43.

[22] Venema HW. Modulation transfer functions of single-slice and dual-slice computed tomography scanners. Med Phys. 1996;23(11):1863-1864.

[23] Löve A, Olsson M-L, Siemund R, et al. Six iterative reconstruction algorithms in brain CT: a phantom study on image quality at different radiation dose levels. Br J Radiol. 2013;86:20130388.

[24] Dodge CT, Tamm EP, Cody DD, et al. Performance evaluation of iterative reconstruction algorithms for achieving CT radiation dose reduction - a phantom study. J Appl Clin Med Phys. 2016;17(2):511-531.

[25] Anam C, Haryanto F, Widita R, et al. An investigation of spatial resolution and noise in reconstructed CT images using iterative reconstruction (IR) and filtered back-projection (FBP). J Phys Conf Series. 2019;1127:012016.

[26] Sanders J, Hurwitz L, Samei E. Patient-specific quantification of image quality: An automated method for measuring spatial resolution in clinical CT images. Med Phys. 2015;43(10):5330-5338. 
[27] Mori I, Machida Y. Deriving the modulation transfer function of CT from extremely noisy edge profiles. Radiol Phys Technol. 2009;2(1):22-32.

[28] Garayoa J, Castro P. A study on image quality provided by a kilovoltage cone-beam computed tomography. J Appl Clin Med Phys. 2013;14(1):239-257.

[29] Boone JM, Seibert JA. An analytical edge spread function model for computer fitting and subsequent calculation of the LSF and MTF. Med Phys. 1994;21(1):1541-1545.

[30] CIRS. AAPM CT Performance Phantom Model 610. http://www.cirsinc.com/wp-content/uploads/2019/04/610-DS-120418.pdf

[31] Sun Nuclear. CT ACR 464 Phantom. https://www.sunnuclear.com/documents/datasheets/gammex/CTACRPhantom_D013118.pdf

[32] The Phantom Laboratory. Catphan ${ }^{\circledR} 500$ and 600 Manual. https://imagingequipment.co.uk/wp-content/uploads/2017/10/Catphan500600-Manual.pdf

[33] Anam C, Budi WS, Fujibuchi T, et al. Validation of the tail replacement method in MTF calculations using the homogeneous and non-homogeneous edges of a phantom. J Phys Conf Series 2019;1248:012001.

[34] Yin FF, Giger ML, Doi K. Measurement of presampling modulation transfer function of film digitizers using a curve fitting technique. Med Phys. 1990;17(6):962-966.

[35] Anam C, Fujibuchi T, Toyoda T, et al. An investigation of a CT noise reduction using a modified of wiener filtering-edge detection. $\mathrm{J}$ Phys Conf Series. 2019;1217:012022.

[36] Ohkubo M, Wada S, Matsumoto T, Nishizawa K. An effective method to verify line and point spread functions measured in computed tomography. Med Phys. 2006;33(8):2757-2764.

[37] Hsieh J. Computed tomography: principles, design artifacts, and recent advances. Bellingham: SPIE; 2003. 